\title{
Total polyphenol intake and breast cancer risk in the Seguimiento Universidad de Navarra (SUN) cohort
}

\author{
Itziar Gardeazabal ${ }^{1,2} \dagger$, Andrea Romanos-Nanclares ${ }^{1} \dagger$, Miguel Ángel Martínez-González ${ }^{1,3,4,5}$, \\ Rodrigo Sánchez-Bayona ${ }^{1,2}$, Facundo Vitelli-Storelli ${ }^{6}$, José Juan Gaforio ${ }^{7,8}$, José Manuel \\ Aramendía-Beitia ${ }^{2}$ and Estefanía Toledo ${ }^{1,3,4_{*}}$ \\ ${ }^{1}$ Department of Preventive Medicine and Public Health, University of Navarra, C/Irunlarrea 1, 31008 Pamplona, Spain \\ ${ }^{2}$ Department of Oncology, University of Navarra, University of Navarra Clinic, Avda Pio XII 36, 31008 Pamplona, Spain \\ ${ }^{3}$ Centro de Investigación Biomédica en Red Área de Fisiopatología de la Obesidad y la Nutrición (CIBEROBN), Av. Monforte de \\ Lemos 3-5, Pabellón 11, Planta 0, 28029 Madrid, Spain \\ ${ }^{4}$ IdiSNA, Navarra Institute for Health Research, C/Irunlarrea 3, 31008 Pamplona, Spain \\ ${ }^{5}$ Department of Nutrition, Harvard T.H. Chan School of Public Health, 677 Huntington Avenue, Boston, MA O2115, USA \\ ${ }^{6}$ Instituto de Biomedicina (IBIOMED), Universidad de León, 24071 Leon, Spain \\ ${ }^{7}$ Center for Advanced Studies in Olive Grove and Olive Oils, Agrifood Campus of International Excellence (ceiA3), University \\ of Jaén, Campus Las Lagunillas s/n, 23071 Jaén, Spain \\ ${ }^{8}$ Centro de Investigación en Red de Epidemiología y Salud Pública (CIBERR-ESP), Instituto de Salud Carlos III, Av. Monforte de \\ Lemos 3-5, Pabellón 11, Planta 0, 28029 Madrid, Spain
}

(Submitted 15 July 2018 - Final revision received 11 November 2018 - Accepted 13 December 2018; First published online 12 February 2019)

\begin{abstract}
Polyphenols are a wide family of phytochemicals present in diverse foods. They might play a role in cancer development and progression. In vivo and in vitro studies have suggested beneficial properties and potential mechanisms. We aimed to evaluate the association between total and main classes of polyphenol intake and breast cancer (BC) risk in the Seguimiento Universidad de Navarra project - a prospective Mediterranean cohort study. We included 10713 middle-aged, Spanish female university graduates. Polyphenol intake was derived from a semi-quantitative FFQ and matching food consumption data from the Phenol-Explorer database. Women with self-reported BC were asked to return a copy of their medical report for confirmation purposes; death certificates were used for fatal cases. Cox models were fitted to estimate multivariable-adjusted hazard ratios (HR) and $95 \%$ CI for the association between tertiles (T) of polyphenol intake and BC. After 10.3 years of median follow-up, 168 probable incident BC cases were identified, out of which 100 were confirmed. We found no association between polyphenol intake and the overall BC risk. Nevertheless, we observed a significant inverse association between total polyphenol intake and $\mathrm{BC}$ risk for postmenopausal women, either for probable or only for confirmed cases $\left(\mathrm{HR}_{\mathrm{T} 3} v\right.$. T1 $\left.0 \cdot 31\left(95 \% \mathrm{CI} 0 \cdot 13,0 \cdot 77 ; P_{\text {trend }}=0 \cdot 010\right)\right)$. Also, phenolic acid intake was inversely associated with postmenopausal BC. In summary, we observed no significant association between total polyphenol intake and BC risk. Despite a low number of incident BC cases in our cohort, higher total polyphenol intake was associated with a lower risk of postmenopausal BC.
\end{abstract}

Key words: Polyphenols: Breast cancer: Postmenopausal breast cancer: Cohort studies: Seguimiento Universidad de Navarra project

Overall, there will be about $2 \cdot 1$ million incident breast cancer (BC) cases diagnosed in 2018. This disease is the most frequently diagnosed cancer in many countries (154 of 185) and is also the leading cause of cancer death in over 100 countries $^{(1)}$. According to 2015 Spanish statistics, BC is the most commonly diagnosed cancer among women ${ }^{(2)}$. Despite the recent data suggesting a decrease in the death rate due to $\mathrm{BC}$, the high prevalence of this disease highlights an unmet need for prevention efforts, which include the promotion of lifestyle factors. Thus, preventive strategies that may help tackle this condition are of great importance from a public health perspective.

One lifestyle factor that has been suggested to be related to $\mathrm{BC}$ is diet. Particularly, previous literature has described a potential association between adherence to the Mediterranean dietary pattern and a reduction in the risk of developing $\mathrm{BC}^{(3)}$.

Abbreviations: BC, breast cancer; SUN, Seguimiento Universidad de Navarra.

* Corresponding author: E. Toledo, email etoledo@unav.es

$\dagger$ Both first and second authors contributed equally to this work. 
Among others, the Mediterranean diet is characterised by a high consumption of fresh fruits and vegetables, nuts and extravirgin olive oil ${ }^{(4)}$. All these foods share in common a high content of polyphenols ${ }^{(5)}$.

Polyphenols are a wide and diverse family of more than 8000 natural substances present in plant foods (cereals, vegetables, fruits, nuts, legumes, etc.) and beverages (wine, tea, coffee, cocoa, etc.) characterised by a structure of at least one aromatic ring with one or more hydroxyl groups attached with a large variability $^{(6)}$. They are considered as responsible for some of the properties of these products, such as their sensory and nutritional qualities. Even though they are not essential for short-term well-being as it is the case for vitamins, they have shown favourable effects on the incidence of chronic diseases including cancer ${ }^{(6)}$. Over the last years, in vitro and in vivo studies have demonstrated bioactivity for many individual polyphenols which includes specific and non-specific properties such as their antioxidant effects, cell cycle arrest, apoptosis stimulation, autophagy promotion, anti-inflammatory effects, anti-oestrogenic mechanisms, aromatase inhibiting properties, human epidermal growth factor receptor 2 (HER2) downregulation and plasma membrane receptors modulation, reduced angiogenesis, reversal of glycolytic metabolism, epigenetic modifications, BC stem cell control, epithelial mesenchymal transition interruption and interference with cancer progression ${ }^{(5,7,8)}$

Several prospective cohort studies assessed the association between single polyphenol subtypes and the risk of $\mathrm{BC}^{(9,10)}$. Nevertheless, evidence on total polyphenol intake and the risk of $\mathrm{BC}$ in prospective studies is scarce ${ }^{(11)}$. In addition, dietary sources of polyphenol intake may vary across different populations. This is why we aimed to assess whether total polyphenol intake and also intake of main polyphenol subtypes were associated with $\mathrm{BC}$ risk in a Mediterranean cohort of university graduates ('Seguimiento Universidad de Navarra' (SUN) project).

\section{Methods}

\section{Study population}

The 'SUN' project (Universidad of Navarra follow-up project) is an ongoing, multipurpose and prospective cohort with open enrolment, which started in December 1999 and includes more than 22000 Spanish university graduates. It aims to assess different associations between diet and lifestyles and the incidence of several chronic diseases and mortality. The study design, methods and cohort profile have been published in detail elsewhere $^{(12)}$. Until 1 December 2016, a total of 22564 participants had completed a baseline questionnaire, from which 13843 were female participants. As we needed at least one of the biennial follow-up questionnaires, we included in the analysis women recruited before 1 March 2014 ( $n$ 13 645).

For the analysis, we excluded women without follow-up ( $n$ 1286, retention rate 91\%), prevalent $\mathrm{BC}$ cases ( $n$ 102), women with a daily energy intake out of the pre-defined limits (below 2092 or beyond $14644 \mathrm{~kJ} / \mathrm{d}$ ) ( $n$ 1345), and those who attained menopause before 35 years of age ( $n$ 199). Finally, our sample size was 10713 women (Fig. 1).

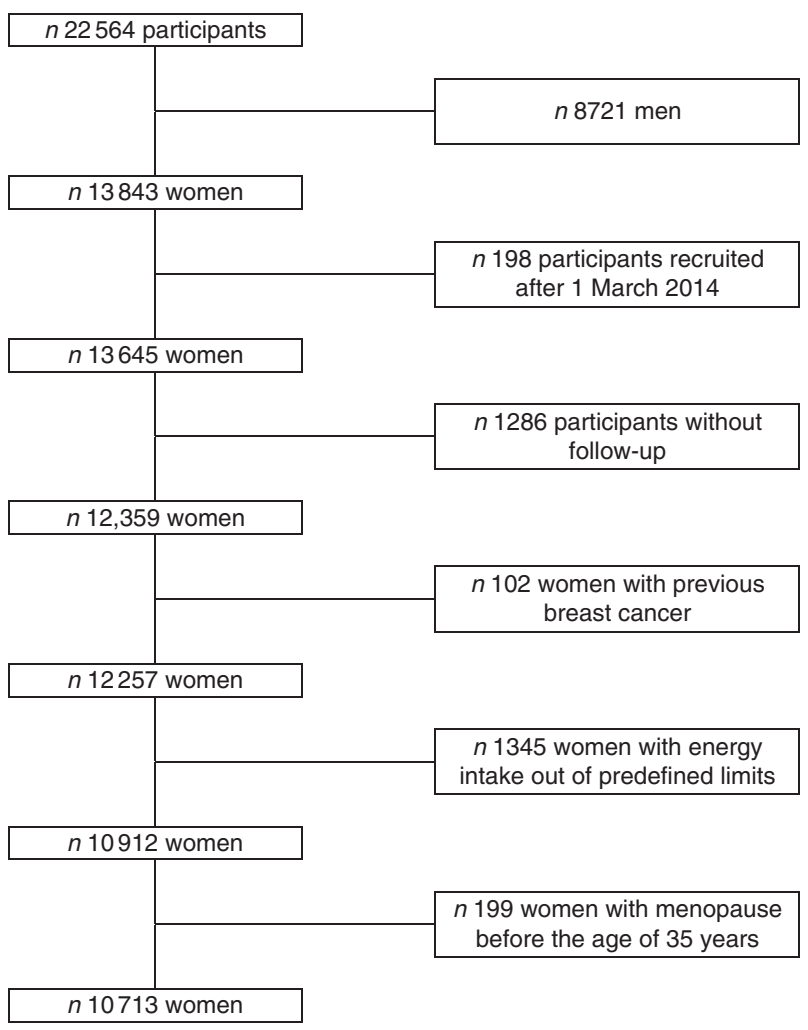

Fig. 1. Flowchart of participants recruited in the Seguimiento Universidad de Navarra project, 1999-2016.

Regarding the informed consent of potential participants, we duly informed these potential candidates of their right to refuse to participate in the SUN study or to withdraw their consent to participate at any time without reprisal, according to the principles of the Declaration of Helsinki. Special attention was given to the specific information needs of individual potential candidates as well as to the methods used to deliver their information and the feedback that may receive in the future from the research team. After ensuring that the candidate had understood the information, we sought their potential freely given informed consent, and their voluntary completion of the baseline questionnaire. These methods were considered by our Institutional Review Board as to imply an appropriately obtained informed consent.

\section{Polyphenol intake and dietary assessment}

Information on diet was collected from a previously validated 136-item FFQ at baseline ${ }^{(13)}$. Consumption frequencies were grouped into nine categories (never or seldom; 1-3 times/ month; once weekly; 2-4 times/week; 5-6 times/week; once daily; 2-3 times/d; 4-6 times/d; 6 or more times per d) according to the typical portion size included for each item. We multiplied typical portion sizes by consumption frequency for each food item to calculate the daily food consumption. For different foods which were assessed together as one single item of the FFQ, we applied a weighted average using weights according to the typical relative frequency of consumption in the Spanish population ${ }^{(14)}$. 
Data on the polyphenol content in foods were collected from the Phenol-Explorer database (www.phenol-explorer.eu) ${ }^{(15)}$ and expressed in $\mathrm{mg} / 100 \mathrm{~g}$ for solid foods and oils or $\mathrm{mg} / 100 \mathrm{ml}$ for beverages. Certain polyphenols are present in foods as aglycone's equivalents which refer to the core flavonoid, phenolic acid, lignan or stilbene, without the combined sugars or polyols.

The data used to estimate polyphenol intake represent the results of normal-phase HPLC for most phenolic compounds. Chromatography following basic or acid hydrolysis was applied for cereals and seeds (lignans in all foods, ellagic acid in walnuts and hydroxycinnamic acids in cereals, legumes and olives). Reverse phase HPLC was used for proanthocyanidins when values were not available by normal phase chromatography ${ }^{(16)}$.

The accordance between food items in the FFQ and the Phenol-Explorer database was evaluated with the following steps: (1) foods with solely traces or without polyphenols were removed, (2) if foods were not included in Phenol-Explorer (leek, thistle and honey), we used the US Department of Agriculture database $^{(17)}$, (3) items on the FFQ comprising more food components such as recipes and processed foods were divided according to their ingredients, (4) retention factors from PhenolExplorer were used to consider the impact of food processing and cooking on polyphenol content values ${ }^{(18,19)}$ and (5) several products containing refined wheat flour were introduced in the list of foods (i.e. pizza, pastries) and polyphenol content was obtained from their wheat flour contents.

Finally, we calculated daily food consumption for the items included in the FFQ and matched this information with PhenolExplorer database on polyphenol content of each reported food (April 2018). Total polyphenol intake was calculated as the sum of all individual classes of polyphenol intake (flavonoids, lignans, stilbenes, phenolic acids, alkylphenols, tyrosols, hydroxybenzaldehydes, hydroxybenxoketones, hydroxycoumarins and methoxyphenols) from all food sources found as stated in this process. We also calculated dietary intake of specific major subclasses of polyphenols, concretely, flavonoids, lignans, stilbenes and phenolic acids ${ }^{(16)}$

\section{Ascertainment of incident breast cancer cases}

Incident cases of $\mathrm{BC}$ were defined as the primary end point for this work. Participants completed follow-up questionnaires biennially in which they were asked to report incident $\mathrm{BC}$ cases. If a participant self-reported an incident case of $\mathrm{BC}$, this participant was asked for a medical record that included this diagnosis, and the diagnosis was confirmed by a trained oncologist. When the medical record to confirm the diagnosis was not yet available, $\mathrm{BC}$ cases were considered for the analyses as probable incident cases. Subjects' next of kin reported fatal cases to our research team. For participants lost to followup or with unknown causes of death, we consulted the National Death Index to identify deceased cohort members and to obtain the cause of death. When the cause of death in the official death certificate was $\mathrm{BC}$, we considered this as a confirmed case.

We stratified our analyses by pre- or postmenopausal BC. Information on age at menopause was collected in the baseline questionnaire and updated after 16 years of follow-up. In the study population, for women lacking information on age at menopause, we established postmenopausal status according to the 75 th percentile of age at menopause (52 years of age) ${ }^{(20)}$. When assessing premenopausal BC as outcome, we excluded those women who reported having had menopause before study inception and considered time at risk from baseline to age of menopause or date of turning 52 years, whichever occurred first. When assessing postmenopausal $\mathrm{BC}$ as outcome, we considered women to be at risk if they were postmenopausal at baseline. We also took into consideration for postmenopausal BC risk ascertainment the observation time of initially premenopausal women of whom we knew they had attained menopausal at a certain time during follow-up - started counting time at risk from that point on - or once they turned 52 years during follow-up.

\section{Evaluation of covariates}

We used the Spanish food composition tables to derive the nutrient composition of diet ${ }^{(21,22)}$. From the baseline questionnaire, we also obtained the information about participants' anthropometric measures, lifestyles, medical history and sociodemographic. Physical activity was assessed with a previously validated questionnaire ${ }^{(23)}$. We estimated metabolic equivalents (MET) for each participant to obtain MET-h/week scores. We had previously validated the accuracy of self-reported weight and height for the calculation of BMI in a subsample of this cohort ${ }^{(24)}$.

\section{Statistical analysis}

Total and individual classes of polyphenol intake were adjusted for total energy intake using the residual method ${ }^{(25)}$ and participants were grouped into tertiles.

We described baseline characteristics of our participants with means and standard deviations for continuous variables and percentages for categorical variables across tertiles of total polyphenol intake.

Person-years of follow-up were calculated for each participant from the date of completion of the baseline questionnaire to the date of $\mathrm{BC}$ diagnosis, the date of death, or the date of return of the last follow-up questionnaire, whichever occurred first. To assess the risk of BC by tertiles of total and individual classes of polyphenol intake (flavonoids, lignans, stilbenes and phenolic acids) ${ }^{(16)}$, we ran Cox proportional hazard regression models with updated diet and covariates. Results are expressed as hazard ratios (HR) and $95 \% \mathrm{CI}$, considering the lowest tertile as the reference category and with age as underlying time variable. In an attempt to control for potential confounding factors, which were selected based on previous literature, we used successive degrees of adjustment: (1) adjustment for age as underlying time variable and calendar year of recruitment in the cohort as stratification variable, (2) additional adjustment for height (continuous), years at university (continuous), number of relatives with history of $\mathrm{BC}$ (three categories), smoking status (never smoker, former smoker and current smoker), physical activity (MET-h/week, continuous), alcohol intake (g/d, continuous), BMI (three categories), age of menarche (five categories), age of menopause (three categories), number of 
pregnancies of more than 6 months (continuous), pregnancy before the age of 30 years (dichotomous), lifetime breastfeeding (continuous), use of hormone replacement therapy (dichotomous) and its duration (continuous) and (3) additional adjustment for diabetes (dichotomous), total energy intake $(\mathrm{kJ} / \mathrm{d}$, continuous) and adherence to the traditional Mediterranean diet (continuous) ${ }^{(26)}$. In postmenopausal women, we additionally adjusted for the time between recruitment and menopause.

We conducted tests of linear trend, assigning to each category of the total polyphenol intake its tertile-specific median and using the resulting variable as continuous in the abovementioned models.

We checked the proportional hazard assumption with the Grambsch-Therneau test of the scaled Schoenfeld residuals ${ }^{(27)}$ and introducing polyphenol intake as a time-varying covariate in the model.

We also calculated the main sources of polyphenol intake as well as the main sources of variability in polyphenol intake in our cohort for both individual foods and food groups (online Supplementary Table S1).

To calculate the contribution of each food item (or group of them) to the between-person variability in polyphenol intake, we conducted nested regression analyses after a stepwise selection algorithm. The contribution of each food group is shown in the cumulative $R^{2}$ change. Furthermore, we estimated their contribution related to total polyphenol intake as the mg consumed from each food group divided by the total polyphenol intake (\%).

Considering that the overall risk of BC in the SUN project was $1.5 \%$ and that we had 3571 women in each tertile, our sample size would allow us to detect absolute risk differences of $0.75 \%$ in $\mathrm{BC}$ between extreme tertiles with a statistical power of $80 \%$ and assuming a two-sided $\alpha$ error of $5 \%$.

All $P$ values were two tailed and a $P$ value $<0.05$ was deemed as statistically significant. All analyses were performed with Stata/SE $15 \cdot 0$.

\section{Results}

Median polyphenol intake was 662 (interquartile range: 482$893) \mathrm{mg} / \mathrm{d}$. Table 1 shows the main characteristics of the 10713 women included in our cohort according to the tertiles of polyphenol intake. We observed that women included in the third tertile were older, more physically active, had a higher alcohol intake, the percentage of pregnancies before 30 years old was higher and consisted of a higher proportion of current smokers and a higher percentage of premenopausal women. Characteristically, we observed a higher time of exposure to hormone replacement therapy for women in the lowest tertile of polyphenol intake. The mean age of the participants at recruitment was 34.7 (SD 10.6) years.

\section{Total polyphenol intake and risk of breast cancer}

During a median follow-up time of $10 \cdot 3$ years and 105847 person-years at risk, 168 probable incident cases of $\mathrm{BC}$ were registered, out of which 100 were confirmed.
Regarding total polyphenol intake and probable BC cases, we found no significant association between total polyphenol intake and overall BC risk, neither for confirmed nor for probable cases (Table 2 and online Supplementary Table S2). When we stratified according to menopausal status, we observed that postmenopausal women within the highest tertile of total polyphenol intake showed a significantly lower risk of $\mathrm{BC}$ when probable cases were considered $\left(\mathrm{HR}_{\mathrm{T} 3} v\right.$. T1 $0 \cdot 35 ; 95 \%$ CI 0.17, $0.73 ; P_{\text {for trend }}=0.005$ ) (online Supplementary Table S3) or when we considered only confirmed cases of BC (Table 3 ) with an HR of $0.31\left(95 \%\right.$ CI $\left.0.13,0.77 ; P_{\text {for trend }}=0.010\right)$ for the highest $v$. the lowest third of total polyphenol intake.

No significant associations were observed for premenopausal women (Table 3 and online Supplementary Table S3).

The contributions of different foods to the variability of total polyphenols intake are shown in Table 4. Individual foods that most contributed to total polyphenol intake were coffee (17.08\%), chocolate $(13.52 \%)$ and cherries $(11.78 \%)$ and to total polyphenol variability cherries, chocolate and apples. On the other hand, when we considered food groups - defined in online Supplementary Table S1 - those that contributed most to total polyphenol intake were fruits $(34 \cdot 13 \%)$, decaffeinated and caffeinated coffee (21.69\%) and chocolate (13.52\%) and those that contributed most to total polyphenol variability were fruits, chocolate and decaffeinated and caffeinated coffee (Table 5).

\section{Individual classes of polyphenols and risk of breast cancer}

When we assessed specifically the association between flavonoids, stilbenes, lignans and phenolic acids (Fig. 2), we observed a significant inverse association between phenolic acid intake and postmenopausal BC. None of the other classes of polyphenols was associated with total, premenopausal or postmenopausal BC.

\section{Discussion}

In this prospective cohort study, we found no overall association between total polyphenol intake and BC risk. By menopausal status, we observed an inverse association between total polyphenol intake and postmenopausal BC risk, even after adjustment for potential confounders. As far as specific classes of polyphenols are concerned, we observed an inverse association between phenolic acids intake and postmenopausal BC risk. No further associations were observed for other subclasses of polyphenols.

To our knowledge, there is no representative study of the Spanish population in which average polyphenol intake has been estimated. In the Prevención con Dieta Mediterránea (PREDIMED) trial, conducted in Spain, in which people at high cardiovascular risk were recruited, mean polyphenol intake was 820 (sD 323) mg/d $\mathrm{d}^{(28)}$. In the European Prospective Investigation into Cancer and Nutrition study, Zamora-Ros et al. ${ }^{(29)}$ reported a median total polyphenol intake for the Mediterranean countries in Europe of $1011 \mathrm{mg} / \mathrm{d}$, which was the lowest in their study, in comparison with non-Mediterranean and UK health-conscious 
Table 1. Baseline characteristics from female participants in the Seguimiento Universidad de Navarra project according to tertiles of polyphenol intake, 1999-2014

(Mean values, standard deviations and percentages)

\begin{tabular}{|c|c|c|c|}
\hline \multirow[b]{2}{*}{ Variables } & \multicolumn{3}{|c|}{ Tertiles of polyphenol intake } \\
\hline & Tertile 1 & Tertile 2 & Tertile 3 \\
\hline Range (mg/d) & $<566 \cdot 0$ & $566 \cdot 0$ to $<800 \cdot 28$ & $800 \cdot 29$ \\
\hline$n$ & 3571 & 3571 & 3571 \\
\hline \multicolumn{4}{|l|}{ Age (years) } \\
\hline Mean & 31.9 & $34 \cdot 8$ & 37.6 \\
\hline SD & $9 \cdot 6$ & $10 \cdot 4$ & $11 \cdot 0$ \\
\hline \multicolumn{4}{|l|}{$\operatorname{BMI}\left(\mathrm{kg} / \mathrm{m}^{2}\right)$} \\
\hline Mean & $22 \cdot 0$ & $22 \cdot 2$ & $22 \cdot 4$ \\
\hline SD & 3.0 & 3.0 & $3 \cdot 1$ \\
\hline \multicolumn{4}{|l|}{ Physical activity (MET/week) } \\
\hline Mean & 22.5 & $24 \cdot 2$ & $26 \cdot 2$ \\
\hline SD & $19 \cdot 2$ & $19 \cdot 7$ & $21 \cdot 8$ \\
\hline \multicolumn{4}{|l|}{ Total energy intake $(\mathrm{kJ} / \mathrm{d})$} \\
\hline Mean & 10012 & 9192 & 9614 \\
\hline SD & 2347 & 2343 & 2435 \\
\hline \multicolumn{4}{|l|}{ Alcohol intake $(\mathrm{g} / \mathrm{d})$} \\
\hline Mean & $3 \cdot 3$ & $4 \cdot 2$ & 4.6 \\
\hline SD & 4.9 & 5.9 & $6 \cdot 7$ \\
\hline \multicolumn{4}{|l|}{ Years at university } \\
\hline Mean & 4.7 & 4.9 & 4.9 \\
\hline SD & 1.3 & 1.3 & 1.4 \\
\hline \multicolumn{4}{|l|}{ Height $(\mathrm{cm})$} \\
\hline Mean & 164 & 163 & 163 \\
\hline SD & 6 & 6 & 6 \\
\hline \multicolumn{4}{|l|}{ Number of pregnancies of more than 6 months } \\
\hline Mean & 0.6 & 0.7 & 0.8 \\
\hline SD & $1 \cdot 1$ & $1 \cdot 1$ & $1 \cdot 2$ \\
\hline \multicolumn{4}{|l|}{ Lifetime breast-feeding (months) } \\
\hline Mean & $6 \cdot 0$ & $6 \cdot 6$ & 6.5 \\
\hline SD & 3.6 & 3.9 & 3.9 \\
\hline \multicolumn{4}{|l|}{ Time of hormone replacement therapy (years) ${ }^{*}$} \\
\hline Mean & 1.5 & $1 \cdot 3$ & $1 \cdot 3$ \\
\hline SD & $2 \cdot 6$ & $2 \cdot 3$ & $2 \cdot 3$ \\
\hline Diabetes (\%) & 1.0 & 1.2 & $1 \cdot 2$ \\
\hline Pregnancies before 30 years of age (\%) & $16 \cdot 4$ & $19 \cdot 2$ & $22 \cdot 2$ \\
\hline \multicolumn{4}{|l|}{ Tobacco (\%) } \\
\hline Never smoker & 55.4 & $48 \cdot 6$ & 45.5 \\
\hline Current smoker & $26 \cdot 2$ & $26 \cdot 0$ & $27 \cdot 1$ \\
\hline Former smoker & $16 \cdot 1$ & $22 \cdot 7$ & 24.7 \\
\hline \multicolumn{4}{|l|}{ Number of relatives with $\mathrm{BC}(\%) \dagger$} \\
\hline 0 & $89 \cdot 2$ & 89.6 & 89.4 \\
\hline 1 & $8 \cdot 7$ & $8 \cdot 7$ & 8.5 \\
\hline 2 & $2 \cdot 1$ & 1.7 & $2 \cdot 2$ \\
\hline \multicolumn{4}{|l|}{ Age of menarche $(\%)$} \\
\hline$<10$ years & $1 \cdot 3$ & $1 \cdot 1$ & $1 \cdot 1$ \\
\hline $10-11$ years & $18 \cdot 6$ & $18 \cdot 8$ & $19 \cdot 8$ \\
\hline $12-13$ years & $54 \cdot 8$ & 55.5 & 53.9 \\
\hline $14-16$ years & $22 \cdot 7$ & $22 \cdot 1$ & $22 \cdot 7$ \\
\hline$>16$ years & $2 \cdot 6$ & $2 \cdot 5$ & 2.5 \\
\hline \multicolumn{4}{|l|}{ Menopausal status (\%) } \\
\hline Premenopausal & $92 \cdot 9$ & $89 \cdot 3$ & $84 \cdot 0$ \\
\hline Postmenopausal & $6 \cdot 1$ & $10 \cdot 7$ & $16 \cdot 0$ \\
\hline Postmenopausal $<50$ years & $65 \cdot 2$ & 62.5 & $68 \cdot 7$ \\
\hline Postmenopausal $50-55$ years & $32 \cdot 0$ & $35 \cdot 7$ & $30 \cdot 6$ \\
\hline Postmenopausal 55 years & $2 \cdot 8$ & 1.8 & 0.7 \\
\hline Mediterranean diet adherence (Trichopoulou score) & 3.44 & $4 \cdot 11$ & 4.72 \\
\hline
\end{tabular}

MET, metabolic equivalents; $\mathrm{BC}$, breast cancer.

* Only for postmenopausal women.

$\dagger$ Information from mother, sister and both grandmothers was collected.

groups. The median intake for population $>30$ years and University graduates was 1150 and $1275 \mathrm{mg} / \mathrm{d}$, respectively. Thus, polyphenol intake in our cohort was lower than that in previous studies.
Evidence on the association between total polyphenol intake and $\mathrm{BC}$ is scarce. Consistent to our results, in the French SUpplémentation en VItamines et Minéraux AntioXydants (SU.VI.MAX) cohort $^{(11)}$, no association was 
Table 2. Confirmed breast cancer cases across tertiles of total polyphenol intake in the Seguimiento Universidad de Navarra project (Hazard ratios (HR) and $95 \%$ confidence intervals)

\begin{tabular}{|c|c|c|c|c|c|c|c|}
\hline & \multicolumn{7}{|c|}{ Tertiles of total polyphenol intake } \\
\hline & \multicolumn{2}{|c|}{ Tertile 1} & \multicolumn{2}{|c|}{ Tertile 2} & \multicolumn{2}{|c|}{ Tertile 3} & \multirow[b]{2}{*}{$P_{\text {for trend }}$} \\
\hline & $\mathrm{HR}$ & $95 \% \mathrm{Cl}$ & $\mathrm{HR}$ & $95 \% \mathrm{Cl}$ & $\mathrm{HR}$ & $95 \% \mathrm{Cl}$ & \\
\hline Cases & \multicolumn{2}{|c|}{30} & \multicolumn{2}{|c|}{32} & \multicolumn{2}{|c|}{38} & \\
\hline Person-year of follow-up & \multicolumn{2}{|c|}{35898} & \multicolumn{2}{|c|}{35360} & \multicolumn{2}{|c|}{34932} & \\
\hline Incidence rate/10 000 person-years & \multicolumn{2}{|c|}{8.4} & \multicolumn{2}{|c|}{$9 \cdot 1$} & \multicolumn{2}{|c|}{$10 \cdot 9$} & \\
\hline Age-adjusted HR & 1 & Ref. & 0.85 & $0.51,1.40$ & 0.85 & $0.52,1.39$ & 0.57 \\
\hline Model $1^{*}$ & 1 & Ref. & 0.86 & $0.52,1.42$ & 0.87 & $0.53,1.42$ & 0.63 \\
\hline Model $2 \dagger$ & 1 & Ref. & 0.88 & $0.53,1.47$ & 0.89 & $0.54,1.49$ & 0.71 \\
\hline
\end{tabular}

Ref., reference.

* Model 1: additional adjustment for height, number of relatives with history of breast cancer (three categories), smoking status (never smoker, former smoker and current smoker), physical activity (metabolic equivalent-h/week, continuous), alcohol intake (g/d, continuous), BMl (three categories), age of menarche (five categories), age of menopause (three categories), number of pregnancies of more than 6 months (continuous), pregnancy before the age of 30 years (dichotomous), months of breast-feeding (continuous), use of hormone replacement therapy (dichotomous) and its duration (continuous) and years at university (continuous).

$\dagger$ Model 2: additional adjustment for diabetes (dichotomous), total energy intake ( $\mathrm{kJ} / \mathrm{d}$, continuous) and Mediterranean diet adherence (continuous).

Table 3. Confirmed breast cancer cases for each tertile of polyphenols in the Seguimiento Universidad de Navarra project among pre- and postmenopausal women

(Hazard ratios (HR) and $95 \%$ confidence intervals)

\begin{tabular}{|c|c|c|c|c|c|c|c|}
\hline & \multicolumn{7}{|c|}{ Tertiles of total polyphenol intake } \\
\hline & \multicolumn{2}{|c|}{ Tertile 1} & \multicolumn{2}{|c|}{ Tertile 2} & \multicolumn{2}{|c|}{ Tertile 3} & \multirow[b]{2}{*}{$P_{\text {for trend }}$} \\
\hline & $\mathrm{HR}$ & $95 \% \mathrm{Cl}$ & $\mathrm{HR}$ & $95 \% \mathrm{Cl}$ & $\mathrm{HR}$ & $95 \% \mathrm{Cl}$ & \\
\hline \multicolumn{8}{|l|}{ Premenopausal breast cancer } \\
\hline$n$ & \multicolumn{2}{|c|}{3298} & \multicolumn{2}{|c|}{3165} & \multicolumn{2}{|c|}{2953} & \\
\hline Cases & \multirow{2}{*}{\multicolumn{2}{|c|}{$\begin{array}{c}14 \\
31353\end{array}$}} & \multirow{2}{*}{\multicolumn{2}{|c|}{$\begin{array}{c}19 \\
28756\end{array}$}} & \multicolumn{2}{|c|}{24} & \\
\hline Person-year of follow-up & & & & & \multirow{2}{*}{\multicolumn{2}{|c|}{$\begin{array}{c}25331 \\
9.5\end{array}$}} & \\
\hline Incidence rate/10 000 person years & \multicolumn{2}{|c|}{4.5} & \multicolumn{2}{|c|}{$6 \cdot 6$} & & & \\
\hline Age-adjusted HR & 1 & Ref. & 1.21 & $0.60,2.42$ & 1.49 & $0.76,2.91$ & 0.24 \\
\hline Model $1^{*}$ & 1 & Ref. & $1 \cdot 21$ & $0.60,2.44$ & 1.50 & $0.76,2.94$ & 0.23 \\
\hline Model 2† & 1 & Ref. & 1.24 & $0.60,2.54$ & 1.69 & $0.84,3.41$ & 0.13 \\
\hline \multicolumn{8}{|l|}{ Postmenopausal breast cancer } \\
\hline$n$ & \multicolumn{2}{|c|}{625} & \multicolumn{2}{|c|}{931} & \multicolumn{2}{|c|}{1289} & \\
\hline Cases & \multicolumn{2}{|c|}{13} & \multirow{2}{*}{\multicolumn{2}{|c|}{$\begin{array}{c}12 \\
5670\end{array}$}} & \multicolumn{2}{|c|}{9} & \\
\hline Person-year of follow-up & \multirow{2}{*}{\multicolumn{2}{|c|}{$\begin{array}{l}3764 \\
34.5\end{array}$}} & & & & & \\
\hline Incidence rate/10000 person-years & & & \multicolumn{2}{|c|}{$21 \cdot 2$} & \multicolumn{2}{|c|}{10.9} & \\
\hline Age-adjusted HR & 1 & Ref. & 0.60 & $0.27,1.31$ & 0.31 & $0.13,0.73$ & 0.008 \\
\hline Model 1ał & 1 & Ref. & 0.72 & $0.32,1.61$ & 0.35 & $0.15,0.84$ & 0.017 \\
\hline Model 2a§ & 1 & Ref. & 0.68 & $0.30,1.57$ & 0.31 & $0.13,0.77$ & 0.010 \\
\hline
\end{tabular}

Ref., reference.

* Model 1: additionally adjusted for height (continuous), number of relatives with history of breast cancer (three categories), smoking status (never smoker, former smoker and current smoker), physical activity (metabolic equivalent-h/week, continuous), alcohol intake ( $\mathrm{g} / \mathrm{d}$, continuous), BMI (three categories), age of menarche (five categories), number of pregnancies of more than 6 months (continuous), pregnancy before the age of 30 years (dichotomous), months of breast-feeding (continuous), use of hormone replacement therapy (dichotomous) and its duration (continuous) and years at university (continuous).

$\dagger$ Model 2: model 1 additionally adjusted for diabetes (dichotomous), total energy intake (kJ/d, continuous) and Mediterranean diet adherence (continuous).

$\$$ Model 1a: model 1 additionally adjusted for time since recruitment (continuous) and age of menopause (three categories).

$\S$ Model 2a: model 2 additionally adjusted for time since recruitment (continuous) and age of menopause (three categories).

observed between total polyphenol intake and BC. Nevertheless, the authors did not report results stratified by postmenopausal status. On the other hand, in our context, the traditional Mediterranean dietary pattern is rich in fruits and vegetables $^{(4)}$ and, thus, in polyphenols. In line with our results, the inverse association between a higher adherence to the traditional Mediterranean dietary pattern and $\mathrm{BC}$ risk seems stronger for postmenopausal $\mathrm{BC}$ than for premenopausal $\mathrm{BC}^{(30)}$.

As far as specific classes of polyphenols are concerned, epidemiological data on phenolic acids is sparse, and to the extent of our awareness, there is only one case-control study that has reported an inverse association between phenolic acids and a hormone-related cancer, concretely, prostate cancer ${ }^{(31)}$. Chlorogenic acids, the most abundant phenolic acids, contained in foods such as coffee and tea, have demonstrated a hefty association with decreased risk of cancer mortality ${ }^{(32)}$ but also improved blood pressure alterations, glucose metabolism and decreased inflammation. Nevertheless, tea is not generally consumed in Southern Europe, albeit major sources of phenolic acids have been stated to be nuts and other foods allied to the Mediterranean diet ${ }^{(33)}$. In our cohort, the main sources of 
Table 4. Sources of variability (cumulative $R^{2}$ ) and main sources (\%) in total polyphenol intake according to each food included in the FFQ

\begin{tabular}{lcc}
\hline Food item & Cumulative $R^{2}$ & $\begin{array}{c}\text { Percentage of total } \\
\text { polyphenol intake }\end{array}$ \\
\hline Cherries and plums & 0.4237 & 11.78 \\
Chocolate & 0.6867 & 13.52 \\
Apple & 0.7618 & 8.78 \\
Regular coffee & 0.8268 & 17.08 \\
Olives & 0.8848 & 5.75 \\
Decaffeinated coffee & 0.9154 & 4.61 \\
Nuts & 0.9336 & 2.76 \\
Swiss chard and spinach & 0.9451 & 2.99 \\
Grapes & 0.9558 & 2.18 \\
Lettuce and endive & 0.9639 & 3.34 \\
\hline
\end{tabular}

Table 5. Sources of variability (cumulative $R^{2}$ ) and main sources (\%) in total polyphenol intake according to food groups

Food group

Fruits

Chocolate

Vegetables

Fruit juices

Nuts

Alcohol

Cereals and legumes

Fats

Pastries
Decaffeinated and caffeinated coffee

Percentage of total Cumulative $R^{2}$ polyphenol intake

$\begin{array}{lr}0.5993 & 34.13 \\ 0.8476 & 13.52 \\ 0.9435 & 21.69 \\ 0.9674 & 12.31 \\ 0.9793 & 4.71 \\ 0.9903 & 3.11 \\ 0.9954 & 2.17 \\ 0.9987 & 4.40 \\ 0.9993 & 2.17 \\ 0.9997 & 1.21\end{array}$

between-person variability in phenolic acids were coffee, fruit, vegetables and nuts (data not shown).

Furthermore, in 2017, Grosso et al. ${ }^{(10)}$ published a large metaanalysis on flavonoid and lignan intake and cancer risk. They found no significant association between total flavonoid intake and $\mathrm{BC}$ risk when they pooled information from prospective cohort studies. Nevertheless, when only prospective cohort studies were pooled in that meta-analysis, a marginally significant inverse association was observed for total lignan intake (the main class of phytoestrogens in Western diets). Also, Rothwell et al. ${ }^{(34)}$ published a review in 2017 where they also described a reduction in $\mathrm{BC}$ risk with soya food consumption higher than $12 \mathrm{~g} / \mathrm{d}$ or 1.5 times/week due to the oestrogenic activity of isoflavones, especially if the intake was in early life. Nevertheless, intake of soya products in Western populations, such as our population, is much lower $(<1 \mathrm{mg} / \mathrm{d})$. When menopausal status was considered, Buck et $a l .{ }^{(35)}$ found an inverse association between lignan intake and postmenopausal $\mathrm{BC}$ in their meta-analysis (seven studies, combined OR: $0.85,95 \%$ CI 0.78, 0.93, $P=0.001$ ), suggesting that the observed result could be due to a possible higher activity of lignans with low oestradiol levels.

In vitro and in vivo trials described different mechanisms by which polyphenols act in cancer prevention and treatment. So hydroxytyrosol may have antioxidants effects and thus protect from DNA damage; resveratrol may inhibit cell proliferation inducing the apoptosis mechanisms; curcumin may exert anti-inflammatory properties; and several polyphenols such as hydroxytyrosol, oleuropein, quercetin and resveratrol may inhibit angiogenesis and cell migration. There are some further mechanisms that may link polyphenol intake and BC risk and more specifically postmenopausal BC risk. Several flavonoids have a similar structure to natural oestrogens, can selectively bind to oestrogen receptor (ER) $\beta$ and, thus, suppress cell proliferation. Hydroxycinnamic acids, the major contributors to total phenolic acid intake, can inhibit cell proliferation, modulate apoptosis and ER $\alpha$ expression, and have antioxidant activity ${ }^{(36)}$. Some polyphenols, as curcumin and olive oil lignans, have shown human epidermal growth factor receptor 2 (HER2) modulation capacity. Finally, flavonoids and resveratrol demonstrated the ability to inhibit aromatase activity. This inhibition decreases the peripheral conversion of androgens to oestrogens, a key mechanism for postmenopausal $\mathrm{BC}^{(5,7,8)}$.

We acknowledge that this work has some limitations. First, we are aware that our statistical power is compromised by the limited number of incident cases of BC. Our cohort is composed largely by young women and most of them are premenopausal, which reduces the number of incident $\mathrm{BC}$ cases. Second, and due to the small number of incident $\mathrm{BC}$ cases, we did not perform a sub-analysis by subtypes of BC cases. Third, dietary information was based on self-reported information. This might have led to some misclassification bias. Nevertheless, the FFQ had been previously validated ${ }^{(13)}$, and the non-differential misclassification would bias our results towards the null value. Fourth, polyphenol content may not be uniform for foods across brands and times. For example, polyphenol content may vary across types of chocolate and coffee over time. This might have led to a non-differential misclassification of the exposure which could have biased our results towards the null. Fifth, the absence of information regarding spices and herbs in the FFQ might have resulted in an underestimation of the polyphenol intake, as, although they are consumed in small amounts, they provide a huge number of polyphenols ${ }^{(37)}$. Sixth, information on $\mathrm{BC}$ incidence also was self-reported. To confirm the accuracy of the information, we asked our participants to send a copy of their medical reports. We run our analyses separately, considering on the one hand those cases that could be confirmed with the medical reports; and on the other hand, all the cases including those that had no medical report confirmation by the day of the analysis. In spite of this, we might have missed some cases of BC. This fact might have reduced the sensitivity to detect incident cases of BC. However, the close follow-up of our participants and the periodic consultation of the National Death Index will have limited the number of missed BC cases. Finally, blood samples from our participants were not available. Therefore, we could not validate the estimated consumption of polyphenols in our cohort.

On the other hand, our study also shows some strengths. It is a dynamic cohort with more than 16 years of follow-up, with $91 \%$ retention rate, and which includes actually 13843 women. Also, we have exhaustive information about the diet of the participants, collected by the FFQ mentioned before. Regarding polyphenol content of foods, we used the Phenol-Explorer database (www.phenol-explorer.eu) ${ }^{(15)}$ to classify them in an accurate manner. Moreover, the FFQ has been previously 
(a)

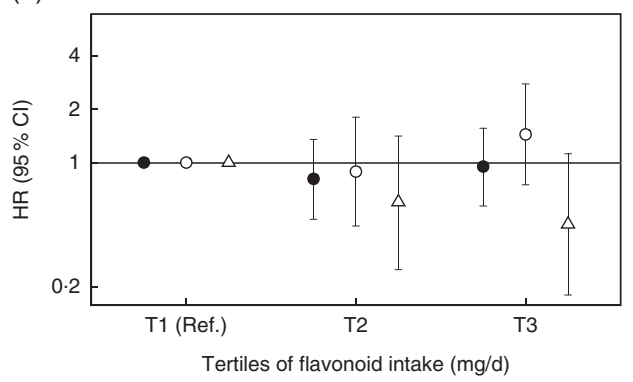

(c)

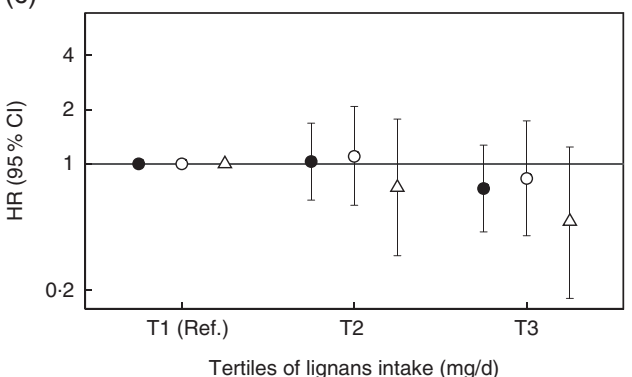

(b)

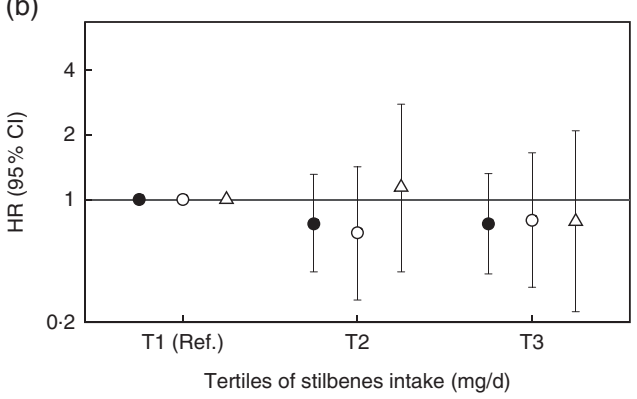

(d)

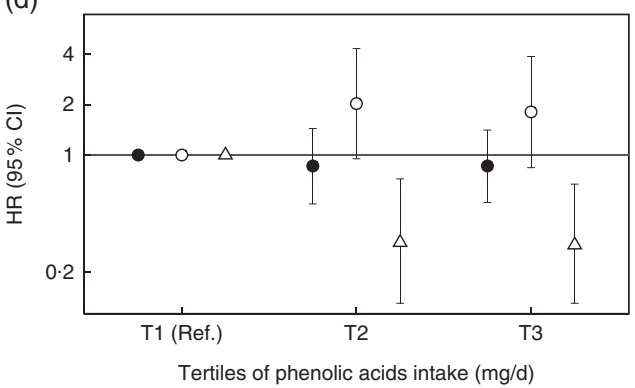

Fig. 2. Hazard ratios $(\mathrm{HR})$ and $95 \%$ confidence intervals for the association between classes of polyphenols intake and risk of breast cancer in the Seguimiento Universidad de Navarra project, 1999-2016. Ref., reference. All tertiles (T) are adjusted for height (continuous), number of relatives with history of breast cancer (three categories), smoking status (never smoker, former smoker and current smoker), physical activity (metabolic equivalent-h/week, continuous), alcohol intake (g/d, continuous), BMI (three categories), age of menarche (five categories), number of pregnancies of more than 6 months (continuous), pregnancy before the age of 30 years (dichotomous), months of breast-feeding (continuous), use of hormone replacement therapy (dichotomous) and its duration (continuous), years at university (continuous), diabetes (dichotomous), total energy intake (kJ/d, continuous) and Mediterranean diet adherence (continuous). For postmenopausal women, we further adjusted for time since recruitment (continuous) and age of menopause (three categories). 0 , Overall women $\left((\mathrm{a}) P_{\text {for trend }}=0.969\right.$, (b) $P_{\text {for trend }}=0.454$, (c) $P_{\text {for }}$ trend $=0.231$ and (d) $\left.P_{\text {for trend }}=0.589\right) ; 0$, premenopausal women ((a) $P_{\text {for trend }}=0.197$, (b) $P_{\text {for trend }}=0.751,(\mathrm{c}) P_{\text {for trend }}=0.606$ and (d) $\left.P_{\text {for trend }}=0.221\right) ; \triangle$, postmenopausal women ((a) $P_{\text {for trend }}=0.239$, (b) $P_{\text {for trend }}=0.515$, (c) $P_{\text {for trend }}=0.126$ and (d) $P_{\text {for trend }}=0.006$ ).

validated and represents the main foods consumed by the study population $^{(38)}$. In previous studies, our group validated the FFQ to assess total polyphenol intake in both clinical and crosssectional studies ${ }^{(39)}$. Moreover, we collected a wide array of potential confounders which have been included in the multivariable analyses, which reduces the room for residual confounding and other potential biases in our results.

\section{Conclusion}

In conclusion, in this Mediterranean cohort study, we observed no significant association between total polyphenol intake and overall BC risk although we found a statistically significant association between total polyphenol intake and the risk of developing a BC among postmenopausal women. Also, among postmenopausal women, we found an inverse association between phenolic acid intake and postmenopausal BC. This is one of the first works evaluating the role of total polyphenol intake and $\mathrm{BC}$ risk. Due to the large in vivo and in vitro evidence, more studies are needed to disentangle the association between polyphenol intake and the risk of overall BC and by subgroups.

\section{Acknowledgements}

The authors are indebted to the participants of the SUN study for their continued cooperation and participation. The authors are also grateful to the members of the Department of Nutrition of the Harvard School of Public Health (W. C. Willett, F. B. Hu and A. Ascherio) who helped us to design the SUN study. The authors also thank other members of the SUN group: A. Alonso, M. T. Barrio López, F. J. Basterra-Gortari, S. Benito Corchón, M. Bes-Rastrollo, J. J. Beunza, S. Carlos Chillerón, L. Carmona, S. Cervantes, J. de Irala Estévez, C. de la Fuente Arrillaga, P. A. de la Rosa, M. Delgado Rodríguez, C. L. Donat Vargas, M. Donázar, A. Fernández Montero, C. Galbete Ciáurriz, M. García López, A. Gea, E. Goñi Ochandorena, F. Guillén Grima, A. Hernández, F. Lahortiga, J. Llorca, C. López del Burgo, A. Marí Sanchís, A. Martí del Moral, N. Martín-Calvo, J. A. Martínez, R. D. Mendonça, J. M. Núñez-Córdoba, P. Pérez de Ciriza, A. M. Pimenta, R. Ramallal, A. Ruiz Zambrana, A. Rico Campà, M. Ruiz-Canela, D. Sánchez Adán, C. Sayón Orea, Z. Vázquez Ruiz and I. Zazpe García.

This work was supported by the Spanish GovernmentInstituto de Salud Carlos III and the European Regional Development Fund (FEDER) (RD 06/0045, CIBER-OBN, grants PI10/02658, PI10/02293, PI13/00615, PI14/01668, PI14/01798, PI14/01764, PI17/01795 and G03/140), the Navarra Regional Government (45/2011, 122/2014 and 41/2016) and the University of Navarra. A. R. N. is supported by the Asociación Española Contra el Cáncer (Spanish Association Against Cancer).

I. G. and E. T. wrote the draft of the manuscript; I. G., A. R.-N., R. S.-B. and E. T. were responsible for data analysis; M. A. M.-G. and E. T. were responsible for conception and design, data acquisition and interpretation. All coauthors revised the 
manuscript critically for important intellectual content and approved the final version to be published.

None of the other authors has conflicts of interest.

\section{Supplementary material}

For supplementary material/s referred to in this article, please visit https://doi.org/10.1017/S0007114518003811

\section{References}

1. Bray F, Ferlay J, Soerjomataram I, et al. (2018) Global cancer statistics 2018: GLOBOCAN estimates of incidence and mortality worldwide for 36 cancers in 185 countries. CA Cancer J Clin 68, 394-424.

2. Galceran J, Ameijide A, Carulla M, et al. (2017) Cancer incidence in Spain, 2015. Clin Transl Oncol 19, 799-825.

3. Toledo E, Salas-Salvadó J, Donat-Vargas C, et al. (2015) Mediterranean diet and invasive breast cancer risk among women at high cardiovascular risk in the PREDIMED trial: a randomized clinical trial. JAMA Intern Med 175, 1752-1760.

4. Trichopoulou A, Martínez-González MA, Tong TY, et al. (2014) Definitions and potential health benefits of the Mediterranean diet: views from experts around the world. $B M C$ Med 12, 112.

5. Gorzynik-Debicka M, Przychodzen P, Cappello F, et al. (2018) Potential health benefits of olive oil and plant polyphenols. Int J Mol Sci 19, E686.

6. Del Rio D, Rodriguez-Mateos A, Spencer JPE, et al. (2013) Dietary (poly)phenolics in human health: structures, bioavailability, and evidence of protective effects against chronic diseases. Antioxid Redox Signal 18, 1818-1892.

7. Losada-Echeberría M, Herranz-López M, Micol V, et al. (2017) Polyphenols as promising drugs against main breast cancer signatures. Antioxidants 6, E88.

8. Mocanu M-M, Nagy P \& Szöllősi J (2015) Chemoprevention of breast cancer by dietary polyphenols. Molecules 20, 22578-22620.

9. Buck K, Zaineddin AK, Vrieling A, et al. (2010) Meta-analyses of lignans and enterolignans in relation to breast cancer risk. Am J Clin Nutr 92, 141-153.

10. Grosso G, Godos J, Lamuela-Raventos R, et al. (2017) A comprehensive meta-analysis on dietary flavonoid and lignan intake and cancer risk: level of evidence and limitations. Mol Nutr Food Res 61, 1600930.

11. Touvier M, Druesne-Pecollo N, Kesse-Guyot E, et al. (2013) Dual association between polyphenol intake and breast cancer risk according to alcohol consumption level: a prospective cohort study. Breast Cancer Res Treat 137, 225-236.

12. Carlos S, De La Fuente-Arrillaga C, Bes-Rastrollo M, et al. (2018) Mediterranean diet and health outcomes in the SUN cohort. Nutrients 10, E439.

13. Martin-Moreno JM, Boyle P, Gorgojo L, et al. (1993) Development and validation of a food frequency questionnaire in Spain. Int J Epidemiol 22, 512-519.

14. Agencia Española de Seguridad Alimentaria y Nutrición (AESAN). Encuesta Nacional de Ingesta Dietética (2011) https://docplayer.es/10558027-Enide-presentacion-n-de-enide2011-encuesta-nacional-de-ingesta-dietetica-tica-espanolaaesan-aesan.html (accessed October 2018).

15. Neveu V, Perez-Jimenez J, Vos F, et al. (2010) PhenolExplorer: an online comprehensive database on polyphenol contents in foods. Database 2010, bap024-bap024.
16. Pérez-Jiménez J, Neveu V, Vos F, et al. (2010) Systematic analysis of the content of 502 polyphenols in 452 foods and beverages: an application of the Phenol-Explorer database. J Agric Food Chem 58, 4959-4969.

17. Bhagwat S, Haytowitz DB \& Wasswa-Kintu S (2014) USDA's expanded flavonoid database for the assessment of dietary intakes. US Departament of Agriculture, Agricultural Research Service, Nutrient Data Laboratory Home Page. http://www. ars.usda.gov/nutrientdata/flav (accessed October 2018).

18. Bognár DA (2002) Tables on weight yield of food and retention factors of food constituents for the calculation of nutrient composition of cooked foods (dishes). http://www.fao.org/ uploads/media/bognar_bfe-r-02-03.pdf (accessed January 2019).

19. Rothwell JA, Medina-Remón A, Pérez-Jiménez J, et al. (2015) Effects of food processing on polyphenol contents: a systematic analysis using Phenol-Explorer data. Mol Nutr Food Res 59, 160-170.

20. Shivappa N, Sandin S, Lof M, et al. (2015) Prospective study of dietary inflammatory index and risk of breast cancer in Swedish women. Br J Cancer 113, 1099-1103.

21. Mataix J (2009) Tablas de Composición de Alimentos (Spanish Food Composition Tables), 5th ed. Granada: University of Granada.

22. Moreiras O, Carbajal A, Cabrera L, et al. (2013) Tablas de composición de alimentos: guia de prácticas (Spanish Food Composition Tables: Practical Guidelines), 16th ed. Madrid: Ediciones Pirámide.

23. Martínez-González MA, López-Fontana C, Varo JJ, et al. (2005) Validation of the Spanish version of the physical activity questionnaire used in the Nurses' Health Study and the Health Professionals' Follow-up Study. Public Health Nutr 8, 920-927.

24. Bes-Rastrollo M, Pérez Valdivieso JR, Sánchez-Villegas A, et al. (2005) Validación del peso e índice de masa corporal autodeclarados de los participantes de una cohorte de graduados universitarios (Validation of self-reported weight and body mass index among participants of a cohort of university graduates). Rev Esp Obes 3, 352-358.

25. Willett W (2013) Nutritional Epidemiology, 3rd ed. New York: Oxford University Press.

26. Trichopoulou A, Costacou T, Bamia C, et al. (2003) Adherence to a Mediterranean diet and survival in a Greek population. $N$ Engl J Med 348, 2599-2608.

27. Royston P \& Lambert PC (2011) Flexible Parametric Survival Analysis Using Stata: Beyond the Cox Model, 1st ed. College Station, TX: Stata Press.

28. Tresserra-Rimbau A, Medina-Remón A, Pérez-Jiménez J, et al. (2013) Dietary intake and major food sources of polyphenols in a Spanish population at high cardiovascular risk: the PREDIMED study. Nutr Metab Cardiovasc Dis 23 , 953-959.

29. Zamora-Ros R, Knaze V, Rothwell JA, et al. (2016) Dietary polyphenol intake in Europe: the European Prospective Investigation into Cancer and Nutrition (EPIC) study. Eur J Nutr 55, 1359-1375.

30. Buckland G, Travier N, Cottet V, et al. (2013) Adherence to the Mediterranean diet and risk of breast cancer in the European prospective investigation into cancer and nutrition cohort study. Int J Cancer 132, 2918-2927.

31. Russo GI, Campisi D, Di Mauro M, et al. (2017) Dietary consumption of phenolic acids and prostate cancer: a case-control study in Sicily, southern Italy. Molecules 22, E2159.

32. Grosso G, Micek A, Godos J, et al. (2016) Coffee consumption and risk of all-cause, cardiovascular, and cancer mortality in 
smokers and non-smokers: a dose-response meta-analysis. Eur J Epidemiol 31, 1191-1205.

33. Godos J, Rapisarda G, Marventano S, et al. (2017) Association between polyphenol intake and adherence to the Mediterranean diet in Sicily, southern Italy. NFS J 8, 1-7.

34. Rothwell JA, Knaze V \& Zamora-Ros R (2017) Polyphenols: dietary assessment and role in the prevention of cancers. Curr Opin Clin Nutr Metab Care 20, 512-521.

35. Buck K, Zaineddin AK, Vrieling A, et al. (2010) Meta-analyses of lignans and enterolignans in relation to breast cancer risk. Am J Clin Nutr 92, 141-153.

36. Rocha LD, Monteiro MC \& Teodoro AJ (2012) Anticancer properties of hydroxycinnamic acids - a review. Cancer Clin Oncol $\mathbf{1}$ 109-121.
37. Pérez-Jiménez J, Neveu V, Vos F, et al. (2010) Identification of the 100 richest dietary sources of polyphenols: an application of the Phenol-Explorer database. Eur J Clin Nutr 64, Suppl. 3, S112-S120.

38. De la Fuente-Arrillaga C, Vázquez Ruiz Z, Bes-Rastrollo M, et al. (2010) Reproducibility of an FFQ validated in Spain. Public Health Nutr 13, 1364-1372.

39. Medina-Remón A, Barrionuevo-González A, Zamora-Ros $\mathrm{R}$, et al. (2009) Rapid Folin-Ciocalteu method using microtiter 96-well plate cartridges for solid phase extraction to assess urinary total phenolic compounds, as a biomarker of total polyphenols intake. Anal Chim Acta 634, 54-60. 